\title{
Testing of an Echelle Spectrometer as a LIBS Detector at Sandia
}

\author{
David P. Baldwin and Daniel S. Zamzow, Ames Laboratory \\ and \\ David K. Ottesen and Howard A. Johnsen, Sandia National Laboratories \\ Ames Laboratory Report IS-5148 \\ April 25, 2001
}

\section{Introduction}

This report summarizes the results of collaborative work performed February 6-9, 2001, at Sandia National Laboratories (Livermore, CA), testing a compact high-resolution spectrometer (CHRS) developed at Ames Laboratory for use as a detection system for the laser-induced breakdown spectroscopy (LIBS) system at Sandia. The CHRS was shipped to Sandia and tested in the LIBS laboratory for the detection of heavy metals introduced into an air exhaust line. The system was also tested at the Combustion Research Facility (CRF) at Sandia for the detection of metals present in the exhaust gas of the Multifuel Combustor, resulting from the introduction of coal and biomass fuels into the combustor.

The LIBS system developed at Sandia has been used as a continuous emissions monitor (CEM) for the detection of toxic metals and other elemental species in stack exhaust gases at a number of combustion, incinerator, and processing facilities. ${ }^{1,2}$ The LIBS system consists of a low-repetition-rate $(<10 \mathrm{~Hz})$, high-pulse-energy $(350 \mathrm{~mJ} /$ pulse $) \mathrm{Nd}$ :YAG laser operating at 1064-nm, for generation of a laser spark used to vaporize and excite analytes present in the gas stream. Optical emission from the laser-induced plasma is collected and delivered using a fiberoptic bundle to a low-resolution spectrometer (a 0.275-m focal-length Acton Research SpectraPro-275) equipped with a time-gateable, intensified CCD array (Princeton Instruments), for the detection of elemental species present in the stack. This system has been used as a CEM to detect metals such as arsenic, beryllium, cadmium, chromium, mercury, and lead at low concentrations in stack exhaust gases. The spectrometer used in the Sandia LIBS system is a low-resolution instrument, which provides only about $0.2-\mathrm{nm}$ resolution. At this spectral resolution, some analytically useful emission lines are unresolved (notably, cadmium and arsenic lines at 228.802-nm and 228.812-nm). Spectral interferences between analyte emission lines, plasma background emission, and emission lines from non-toxic metals present in the exhaust gas (iron and other species) can also be a potential limitation for the LIBS system, using a lowresolution spectrometer. A low-resolution spectrometer requires the use of a time-gateable, intensified CCD to discriminate analyte emission signals from the intense laser-induced plasma background emission that is present immediately after the laser pulse. A CCD detector gate width of a few $\mu \mathrm{s}$, with a delay time of a few $\mu$ s after the laser pulse, is generally used to maximize the signal-to-noise ratio for analyte lines in LIBS experiments. Different delay times and gate widths are often used for different analytes to optimize the detection sensitivity.

The CHRS developed at Ames Laboratory consists of an acousto-optic tunable filter (AOTF, from MVM Electronics) and a 0.4-m focal-length echelle spectrometer. ${ }^{3-5}$ This system is equipped with a standard (unintensified, not time-gateable, back-thinned) CCD array (Hamamatsu). The AOTF acts as an order-sorting bandpass pre-filter for the echelle 
spectrometer by selecting a $\sim 1$-nm bandwidth of optical emission that is introduced into the echelle. The echelle spectrometer provides high-resolution detection of the optical emission, with a spectral resolution that ranges from approximately $0.004-\mathrm{nm}$ at $200-\mathrm{nm}$ to $0.01-\mathrm{nm}$ at 500 -nm (a resolving power of 1:50,000). The AOTF-echelle has been used as a CEM for the detection of metals introduced into an air-inductively coupled plasma (air-ICP) system. For the work discussed in this report, the echelle spectrometer was tested at Sandia to determine its potential as a detection system for LIBS monitoring applications.

\section{Results and Discussion}

During testing of the echelle spectrometer system at Sandia, the AOTF did not function. During the test, the problem was thought to be an AOTF frequency-driver failure (no RF output power). After the test, the problem was determined to be an issue with the parallel port of the laptop computer used to control the AOTF. This computer has been used previously to control the AOTF, but failed during the Sandia test. As a result, a 0.2-m focal-length grating monochromator (ISA) was used as a bandpass pre-filter for the echelle spectrometer rather than the AOTF. This monochromator has a light throughput that is roughly comparable to that of the AOTF, but it must be mechanically scanned to select the wavelength of light that is introduced to the echelle. For the AOTF, the wavelength is selected by applying an RF frequency to the device; any wavelength from 220-450 $\mathrm{nm}$ can be selected in milliseconds by changing the applied RF frequency. This AOTF system has a multi-frequency driver that enables three wavelength intervals to be selected simultaneously, to allow the echelle spectrometer to be used for multi-metals monitoring applications. Unfortunately, due to the problem with the laptop computer, the intended testing of the AOTF-echelle spectrometer for LIBS monitoring was not done during the Sandia site-test. However, substitution of the 0.2-m monochromator for the AOTF allowed the test to continue, and allowed the acquisition of the data presented below for the echelle spectrometer.

The echelle spectrometer was initially tested in the LIBS laboratory for the detection of metals introduced into an air exhaust line system. For some of these tests, a direct comparison of the two systems (the SpectraPro-275 with the Princeton Instruments CCD and the echelle spectrometer with the Hamamatsu CCD) was done by collecting data simultaneously, using a bifurcated fiber-optic bundle to deliver LIBS signals to the two spectrometers. For some tests, the Princeton Instruments CCD was used as the detector on the echelle spectrometer to allow a comparison of the spectrometers using the same detection electronics. The laboratory data presented below were acquired during the first two days of the site-test. At the end of the second day, the LIBS instrumentation was moved to the CRF. On the following day, the two spectrometer systems were used concurrently for the LIBS detection of atomic species present in the exhaust gas of the Multifuel Combustor, resulting from the introduction of coal (Illinois no. 6) and biomass (sugar cane bagasse) fuels.

The light throughput of the SpectraPro-275 is significantly higher than that for the echelle spectrometer system. An example of comparative LIBS data acquired using the two spectrometers (with the Princeton Instruments CCD as the detector) is shown in Figure 1 for lead. Lead was introduced into the air exhaust line at an aerosol concentration of approximately $12 \mathrm{mg} / \mathrm{m}^{3}$. The upper spectrum in Figure 1 is a portion of the $\sim 35-\mathrm{nm}$ spectral window centered on the $\mathrm{Pb}$ (I) 405.78-nm emission line, obtained using the SpectraPro-275. The lower spectrum 
is the $\mathrm{Pb}$ signal detected using the echelle spectrometer. The echelle is a higher resolution spectrometer, so a smaller spectral window is detected. For this comparison, the signal intensity (peak height) detected using the SpectraPro-275 is a factor of almost 200 greater than that for the echelle spectrometer. The echelle is a higher $\mathrm{f} /$ number spectrometer than the SpectraPro-275 (f/8 compared to $\mathrm{f} / 3.8$ ) and there are additional optical components (monochromator used as a bandpass pre-filter and one focusing lens between the monochromator and the echelle), so a lower light throughput is expected for the echelle. Different slit widths $(70 \mu \mathrm{m}$ for the SpectraPro-275 and $25 \mu \mathrm{m}$ for the echelle) were used, and this also contributes to the difference in signal intensity for the two spectra in Figure 1. All of the optical components of the two spectrometer systems must be considered when comparing the detection sensitivity for the two systems.

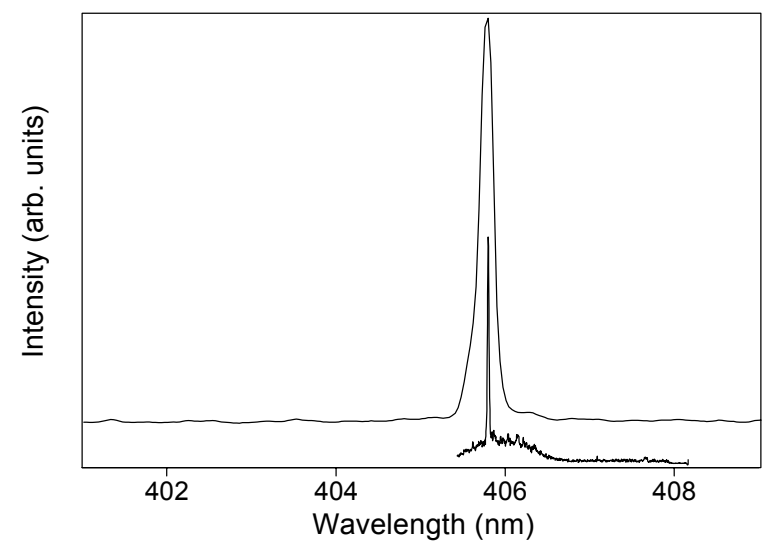

Figure 1. LIBS detection of $12 \mathrm{mg} / \mathrm{m}^{3}$ lead in air, for the $\mathrm{Pb}$ (I) 405.78-nm emission line, using the SpectraPro-275 (upper spectrum) and the echelle spectrometer (lower trace). For this comparison, the Princeton Instruments CCD was used as the detector for both spectrometers. For the echelle spectrum, the intensity scale has been expanded by a factor of 100 .

The high resolution of the echelle spectrometer allows the detection of closely-spaced analyte emission lines, such as the $\mathrm{Cd}$ (I) 228.802-nm and As (I) 228.812-nm line pair. Figure 2 shows LIBS spectra obtained using the echelle spectrometer, for a mixture of cadmium and arsenic in air at aerosol concentrations of approximately $3.3 \mathrm{mg} / \mathrm{m}^{3} \mathrm{Cd}$ and $16.7 \mathrm{mg} / \mathrm{m}^{3}$ As (the upper six spectra in Figure 2). The bottom trace in Figure 2 is an air-ICP reference spectrum for a Cd-As mixture obtained using the AOTF-echelle spectrometer, showing near baseline resolution of these emission lines. For the LIBS spectra in Figure 2, the detected signal is at about the signal-to-noise limit, and the variation in the $\mathrm{Cd} / \mathrm{As}$ intensity ratio evident in the spectra is believed to be due to differences in LIBS excitation of $\mathrm{Cd}$ and As particles during data acquisition (photon- and particle-counting statistics). The resolution for the LIBS Cd-As spectra in Figure 2 is degraded compared to that for the air-ICP spectrum for a number of reasons. A larger echelle entrance slit width $(50 \mu \mathrm{m}$ rather than $25 \mu \mathrm{m})$ was used to increase the LIBS signal intensity. The LIBS spectra were acquired using the Princeton Instruments (intensified) CCD, not the Hamamatsu (unintensified) CCD; some degradation in pixel resolution normally occurs for intensified detectors. Finally, due to differences in the physical geometry of the two detectors (different focal position distances to the CCD chips in the detector mounts), the Princeton Instruments CCD was used on the echelle spectrometer at slightly less-than-optimum focus. That is, for the optical alignment of the echelle spectrometer during the test at Sandia, the 
Princeton Instruments CCD could not be positioned in the focal plane at a location that provided the best resolution.

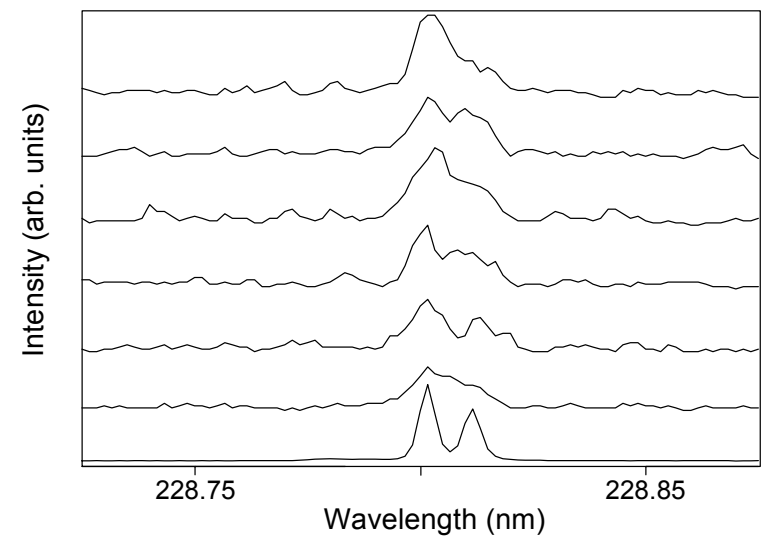

Figure 2. LIBS spectra acquired for a mixture of cadmium and arsenic, for the Cd (I) 228.802-nm and As (I) 228.812-nm emission lines, using the echelle spectrometer (upper six spectra). The lower trace is an air-ICP reference spectrum for a Cd-As mixture.

The Cd-As line pair shown in Figure 2 is unresolvable using the SpectraPro-275 spectrometer. Other closely-spaced analyte lines (such as the Be (II) 313.042- and 313.107-nm lines) were detected and resolved using the echelle spectrometer during laboratory LIBS testing at Sandia. The difference in spectral resolution of the SpectraPro-275 and echelle spectrometers is illustrated in Figure 3, for LIBS spectra acquired at the CRF during the introduction of pulverized coal into the Multifuel Combustor. The spectra show titanium emission lines at 323.45-, 323.66-, 323.90-, and 324.20-nm, for the SpectraPro-275 (upper spectrum) and the echelle spectrometer (lower trace). The spectral resolution for the echelle is about 30 times higher than that for the SpectraPro-275 system (approximately 0.007-nm compared to about 0.2$\mathrm{nm}$ at $323-\mathrm{nm})$. A limited spectral window is detected using the echelle spectrometer $(\sim 1-\mathrm{nm}$ bandpass for the monochromator used as a pre-filter for the echelle); the spectrum displayed in Figure 3 for the SpectraPro-275 is only a portion of the $\sim 35-\mathrm{nm}$ spectral window detected.

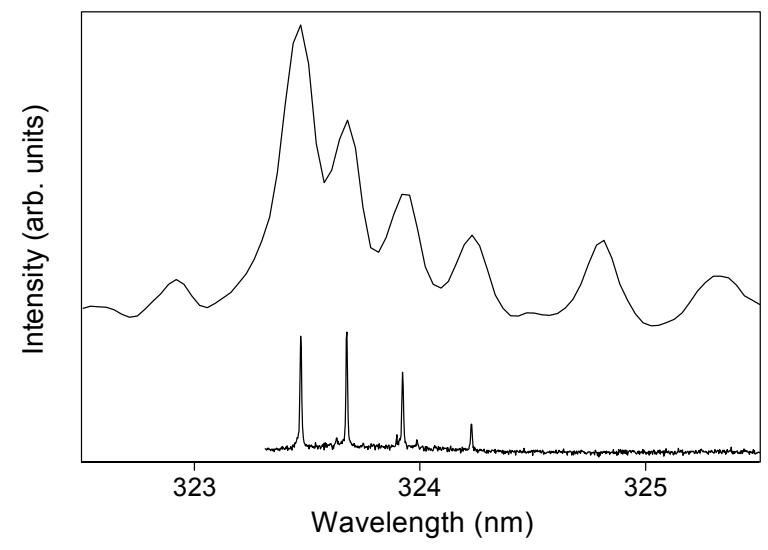

Figure 3. LIBS spectra acquired at the CRF at Sandia, during introduction of coal into the Multifuel Combustor. The spectra show emission lines for titanium present in the coal (Ti (II) 323.45-, 323.66-, 323.90-, and 324.20-nm lines), detected using the SpectraPro-275 (upper spectrum) and echelle spectrometer (lower trace). 
During the introduction of coal and biomass feed materials into the Multifuel Combustor at the CRF, the two spectrometer systems were used concurrently for LIBS detection of metals present in the exhaust gas of the combustor. Spectral data were acquired to identify metals present in the coal and biomass samples. The same group of analytes (including aluminum, calcium, carbon, iron, magnesium, sodium, silicon, and titanium) was detected using the two spectrometers, with the SpectraPro-275 providing higher detection sensitivity and the echelle providing higher detection resolution. During some of the LIBS testing at the CRF, the echelle spectrometer was used as a CEM by acquiring time-scan data, as the operating conditions for the combustor (material feed rate and composition) were varied. An example of this is shown in Figure 4 for the LIBS detection of aluminum and calcium, as the material introduced to the combustor was switched from pulverized coal (Illinois no. 6) to biomass (sugar cane bagasse). In Figure 4, the LIBS signal for calcium (Ca (II) 396.85-nm) is plotted as the upper time-scan trace, and that for aluminum (Al (I) 396.15-nm) is the lower trace. The data points from approximately 565-585 seconds in Figure 4 indicate a period of time that the laser was turned off, while the feed material to the combustor was being switched from coal to biomass. A relatively high standard deviation (about $30 \%$ rsd for the $\mathrm{Al}$ and $\mathrm{Ca}$ signals for coal and greater than $50 \%$ rsd for the biomass data) is observed, despite the use of a signal integration time of five seconds per point for the data in Figure 4. Since a laser repetition rate of $8.5 \mathrm{~Hz}$ was used, each data point represents a LIBS signal averaged over more than 40 laser shots. Much of the fluctuation in the measured signals is attributable to the variation in the material feed rate to the combustor. The average $\mathrm{Al} / \mathrm{Ca}$ intensity ratio for the coal is 4.8 times higher than that for the biomass sample. For the coal data, the standard deviation of the $\mathrm{Al} / \mathrm{Ca}$ intensity ratio is lower than that for either the $\mathrm{Al}$ or Ca signals (17\% rsd compared to about $30 \% \mathrm{rsd}$ ), indicating that the $\mathrm{Al}$ and $\mathrm{Ca}$ signals are correlated $\left(\mathrm{r}^{2}=0.624\right)$. For the biomass data, the standard deviation of the $\mathrm{Al} / \mathrm{Ca}$ intensity ratio is high (54\% rsd), so the $\mathrm{Al}$ and Ca LIBS emission signals are not correlated to any significant degree in this case $\left(r^{2}=0.245\right)$.

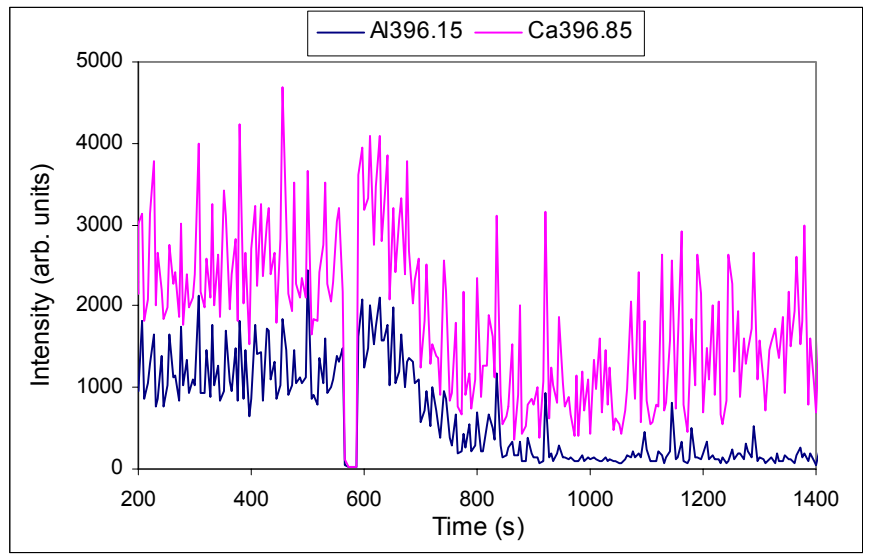

Figure 4. LIBS time-scan monitoring data obtained using the echelle spectrometer, for aluminum and calcium in coal and biomass feed materials introduced into the Multifuel Combustor at the CRF. A spectral window centered on the $\mathrm{Al}$ (I) 396.15-nm and Ca (II) 396.85-nm emission lines was used for data acquisition. The initial data (for points at times from 200 to about 600 seconds) were obtained for coal; the switch from coal to biomass was completed by a point corresponding to about 850 seconds. 


\section{Conclusions}

Some useful information has been obtained regarding the potential use of the echelle spectrometer system for LIBS monitoring applications, despite the AOTF-computer operational problems during the Sandia site-test. Currently, the use of the echelle spectrometer with the LIBS system is not suitable for trace-level analyte detection. This is due, in part, to the lower light throughput of the echelle spectrometer system compared to the SpectraPro-275. The low duty cycle of the LIBS system, which results from the use of a low-repetition-rate (but low-cost and portable) laser, also limits the detection sensitivity achievable using a high-resolution spectrometer. At high analyte concentrations, the echelle spectrometer is able to resolve spectral interferences including the Cd-As line pair at 228.8-nm and other LIBS emission features not resolved using the SpectraPro-275. A definite positive result obtained is the determination that at the high resolution of the echelle spectrometer, time-gating of the CCD detector is not necessary to discriminate analyte spectral signals from the LIBS background emission. The cost of the gated CCD and associated electronics is a significant portion of the cost of the Sandia LIBS system. Incorporation of a low-cost version of the echelle spectrometer for process monitoring applications not requiring trace-level detection could make LIBS a more viable technique where cost is a limiting factor. We hope to have the opportunity to perform additional collaborative work using the AOTF-echelle spectrometer for on-line LIBS monitoring applications, in order to demonstrate the advantage of rapid line-switching (using the AOTF) and simultaneous detection of multiple emission features across the spectral range of the echelle.

\section{References}

1) S. G. Buckley, H. A. Johnsen, K. R. Hencken, and D. W. Hahn, Waste Management 20, $455-$ 462, 2000.

2) D. W. Hahn, W. L. Flower, and K. R. Hencken, Appl. Spectrosc. 51, 1836-1844, 1997.

3) D. P. Baldwin, D. S. Zamzow, D. E. Eckels, and G. P. Miller, in Environmental Monitoring and Remediation Technologies II, T. Vo-Dinh and R. L. Spellicy (Eds.), Proceedings of SPIE Vol. 3853, pp. 213-220, 1999.

4) D. P. Baldwin, D. S. Zamzow, D. E. Eckels, and G. P. Miller, in Environmental Monitoring and Remediation Technologies, T. Vo-Dinh and R. L. Spellicy (Eds.), Proceedings of SPIE Vol. 3534, pp. 478-486, 1999.

5) D. P. Baldwin, D. S. Zamzow, D. E. Eckels, G. P. Miller, R. Wiser, and S. Tao, "Testing of a Continuous Sampling Air-ICP System as a Continuous Emission Monitor at the Diagnostic Instrumentation and Analysis Laboratory, September 12-17, 1999," Ames Laboratory Report IS-5138, October, 1999. 